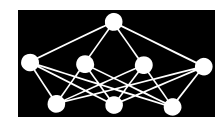

\title{
NEURAL NETWORK BASED IDENTIFICATION OF TRICHODERMA SPECIES
}

\author{
A. Akbarimajd, M. Selseleh Jonban† M. Nooshyar $*$ M. Davari $\eta^{\ddagger}$
}

\begin{abstract}
The genus Trichoderma acts as an important antagonist against phytopathogenic fungi. This paper proposes a software-based identification tool for recognition of different species of Trichoderma. The method uses the morphological features for identification. Morphological-based species recognition is common method for identifying fungi, but regarding the similarity of morphological features among different species, their manual identification is difficult, time-consuming and may bring about faulty results. In this paper it is intended to identify different species of Trichoderma by means of neural network. For this purpose, 14 characteristics are used including 5 macroscopic and 9 microscopic characteristics. After quantifying qualitative features and training a multilayer perceptron neural network with quantified data, 25 species of Trichoderma are recognized by using the network. Totally, identification of Trichoderma species as one useful fungus is achieved by using the trained network.
\end{abstract}

Key words: Trichoderma spp., classification, multilayer perception network, feature quantification

Received: June 14, 2014

DOI: $10.14311 / \mathrm{NNW} .2016 .26 .009$

Revised and accepted: April 19, 2016

\section{Introduction}

The fungal genus Trichoderma (Ascomycetes, Hypocreales) is the most common saprophytic filamentous imperfect fungi that functions as a biocontrol agent for a wide range of economically important aerial and soilborne plant pathogens such as Fusarium, Pythium, Sclerotinia, Rhizoctoniaand Botrytis in crop plants [10, 2]. In addition, Trichoderma has been reported to be effective in promoting plant growth $[12,26]$ and to have the ability to resist against inducement in the plants [14]. Several strains of the genus Trichoderma are being tested as alternatives to

\footnotetext{
*Adel Akbarimajd, Mehdi Nooshyar, Department of Electrical Engineering, Faculty of Engineering, University of Mohaghegh Ardabili, Ardabil, Iran, E-mail: akbarimajd@uma.ac.ir, nooshyar@uma.ac.ir

${ }^{\dagger}$ Mansour Selseleh Jonban - Corresponding author, Young Researchers and Elite Club, Ahar Branch, Islamic Azad University, Ahar, Iran, E-mail: m-selselehjonban@iau-ahar.ac.ir

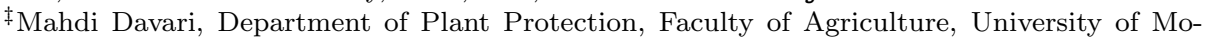
haghegh Ardabili, Ardabil, Iran, E-mail: mdavari@uma.ac.ir
} 
chemical fungicides. The other role of these fungi is converting organic wastes and compost production. These fungi are also able to perform easily and fast the fermentation, decomposition of cellulose, hemicelluloses, lignin and are very useful in compost production [4]. Recently, Some Trichoderma species such as T. viride and T. koningi have been used in biosynthesis of silver nanoparticles and showed the antimicrobial activity in medicine $[8,37]$.

Morphological characteristics and taxonomies of fungal species are useful for many purposes and can be served as useful tools for initial detection of species [30, 31]. As pointed out by many researchers, the major advantages of the morphological identification method are the general applicability of this method to all fungal taxon and its widespread use [36]. With the advent of molecular techniques, rapid progress in gene and genome sequences technologies, DNA based approaches are becoming as a gold standard technique for species recognition in fungi. However, the usefulness of morphological criteria for species recognition and morphological discrimination at species level is important for end users at agriculture, medicine and industry [31].

It is important to identify Trichoderma as a particular fungus that potentially is used to eliminate a specific disease. For example, T. harzianum species has been detected to be very effective in controlling postharvest diseases such as Penicillium expansum Link [3]. Apparently, using other species in this case would be ineffective and exactness of the species should be ensured. Due to the ecological importance of Trichoderma, its application as a biocontrol agent in the field and human activity, it is important to identify its species in a precise and rapid way. Nevertheless, accurate species identification based on morphological characteristics which is manually performed by available identification keys is difficult task and time-consuming even for experts $[10,9]$. In this paper, 25 species of Trichodermaare identified and distinguished using artificial neural networks. For this purpose, morphological data of these fungi will be used which is given in [7].

\section{Nomenclature}

\begin{tabular}{cl}
\hline Symbol & Nomenclature \\
\hline$E_{p}$ & Error for $p$ model \\
$t_{j}^{p}$ & Target output for $p$-th input in $j$-th cell of the output layer \\
$a_{j}^{p}$ & Produced real output for $p$-th input in $j$-th cell of the output layer \\
$\mathbf{r}$ & Length of input vector \\
$\mathbf{s}_{1}$ & Length of output vector \\
$\mathbf{w}$ & Weight vector \\
$\eta$ & Learning rate \\
$\alpha$ & Instantaneous coefficient \\
$\mathbf{b}$ & Bias vector \\
$\mathbf{w}_{i j}(t+1)$ & New weight \\
$\mathbf{w}_{i j}(t)$ & Old weight \\
$\mathbf{b}_{j}(t+1)$ & New bias \\
$\mathbf{b}_{j}(t)$ & Old bias \\
\hline
\end{tabular}




\section{Related works}

Common method for distinguishing fungi is a method which is called synoptic method. In this method, after extracting morphology characters, for identification of species, morphological characteristics are compared with validation identification keys. In [32, 33], this method was used for identifying species of Trichoderma. In [5], an approach was presented for recognizing various species of Phytophthora. This approach is a web-based identification which guides users to answer by progressively selecting existing states on the web. It is obvious that these approaches are not intelligent; thus in this paper an intelligent method is presented. For this purpose, neural network is utilized that has not already been used for recognizing fungi.

In different fields, various works have been reported on classification by artificial neural networks (ANN). For example, in the agriculture, different methods have been employed for classification of wheat, barley, rice and tea seeds [16, 17, 19, 39]. Zapotoczny et al. used probabilistic ANN to classify 11 varieties of wheat (seven winter and four spring) varieties. At first, input data has been normalized to be within $[-1,+1]$ interval and then divided into two groups including learning data and validation data. Subsequently, assuring stochastic nature of cases applied into the ANN, dataset was generated with specified states for each of the 11 investigated species. With this method, Zapotoczny could achieve classification accuracy of $100 \%$ in identification of the wheat varieties.

In the medical researches, applications of classification have been increased in clustering data for rapid diagnostic of illnesses. In diagnosing cardiac arrhythmia, disorders of cardiac rhythm, using the artificial neural networks as a tool, data obtained from human heart rate were firstly classified in two groups of normal and abnormal heart rates. After training ANN by learning data and testing it on the testing data, the network could be employed in diagnosis of the disease $[6,15,27$, $28,29]$. In this field, one can refer to a research conducted by Ozbaya et al. in 2001 where diagnosis of cardiac arrhythmia was done by using of artificial neural network architecture. Afterwards in 2006 they could make use of the network to classify 10 different rhythms for 92 patients (40 males and 52 females) by designing a multilayer perceptron ANN and fuzzy clustering neural network architecture. Then, diagnostic accuracy was increased from $97.8 \%$ in [27] to over $99 \%$ in [29] by combining the neural network and fuzzy technique with wavelet transform.

Zhu et al. proposed an artificial neural network for bacteria classification based on morphometric characteristics [40]. Essentially, manual bacteria classification is a tedious work and often needs abundant correlative data. In the network proposed by Zhu et al., bacteria's morphometric was applied to ANN as input and the network could classify bacteria.

Neural networks have also been employed for classifying proteins. Among most recent works, it can be referred to [25] and [38]. In [25], the authors employed position specific scoring matrices and amino acid properties to provide a technique based on radial basis function networks for identification of efflux proteins. In [38], a novel ab initio predictor of protein enzymatic class was presented. The predictor could classify proteins into one of six classes, only according to their sequences. The network was trained on a large database selected of over 6,000 non-redundant proteins. 
Few papers have been published reporting application of neural networks in fungus classification. Nilson et al. used some spectrometry techniques followed by multivariate analysis and artificial neural networks for classification of species in the genus penicillium [18]. In [24], an artificial neural network was trained based on morphometric data from spores of Pestalotiopsis spp. The network showed identification accuracy not more than $78.8 \%$ of a 16 -species group and $67.7 \%$ of a 19-species group. In [33], in order to classify mycelia of 26 fungal strains belonged to 24 different species, Fourier transform infrared spectroscopy was obtained and comparative artificial neural network analysis was performed. These works have employed combination of neural networks and other techniques for classification purposes. They have not achieved accuracy more than $80 \%$. Moreover, identification of different species of Trichoderma - which is the subject of this paper - is more difficult than genus considered in abovementioned papers. This difficulty will be described in detail in next sections.

\section{Problem description}

Identification of fungi is usually done using one of the several well-illustrated dichotomous keys $[1,23]$. Multi-access keys are very useful for biological agents, especially for non-specialists, as it eliminates necessity of detecting all of the fine descriptions usually found in dichotomous keys [23]. The disadvantages of those printed keys are that they require that the user is able to scan a series of tables of numbers and select those are best fitted to the specimen being examined [31, 34]. In this paper, morphological characteristics are employed for detecting 25 Trichoderma species. For this purpose, 14 characteristics those are common in Trichoderma identification keys, will be used. These characteristics include two categories: macroscopic and microscopic characteristics. The macroscopic characteristics include colony morphology, pigmentation and growth rates of cultures on PDA (Potato Dextrose Agar) medium at $25{ }^{\circ} \mathrm{C}$ after 3 days and Conidia forming time on Agar. Microscopic features include: color, surface, form, length and width of the conidia, shape of phialide, length of phialide, width of phialide at its widest sector and width of phialide's base. Fig. 1 illustrates phenotypic characters of two species of Trichoderma in which images A and D are related to colony appearance, images $\mathrm{B}$ and $\mathrm{E}$ are related to phialide shape, and finally images $\mathrm{C}$ and $\mathrm{F}$ are related to conidia shape [21].

Identification of Trichoderma species based on phenotypic characters by matching these characteristics with valid identification keys is a time-consuming task and may produce inaccurate results when performing manually. As it is impossible to visualize 14 dimensional spaces, in order to graphically illustrate the issue, these species are shown in four 3-dimensional diagrams. In each diagram, each point denotes three different characteristics regardless to other 11 ones. In Fig. 2(a), points are shown in terms of Agar paint, width at the widest sector of the phialide and length of conidia. In Fig. 2(b) shape of phialide, length of phialide and growth rates of colony after three days are selected to show the points. Fig. 2(c) includes width of conidia, shape of conidia and colony morphology as axes of the diagram. Finally, Fig. 2(d) shows width of phialide's base, conidia color and surface of conidia. From these figures, it is obvious that the Trichoderma species are very close 
to each other in characteristics space. Hence, it is very difficult to visually identify them and make an effective classification.

In this paper, it is attempted to identify Trichoderma species by designing a multilayer perceptron network and training it. This network is able to recognize and identify the species of this fungal genus in about a few tenths of a second by just using the morphological characteristics

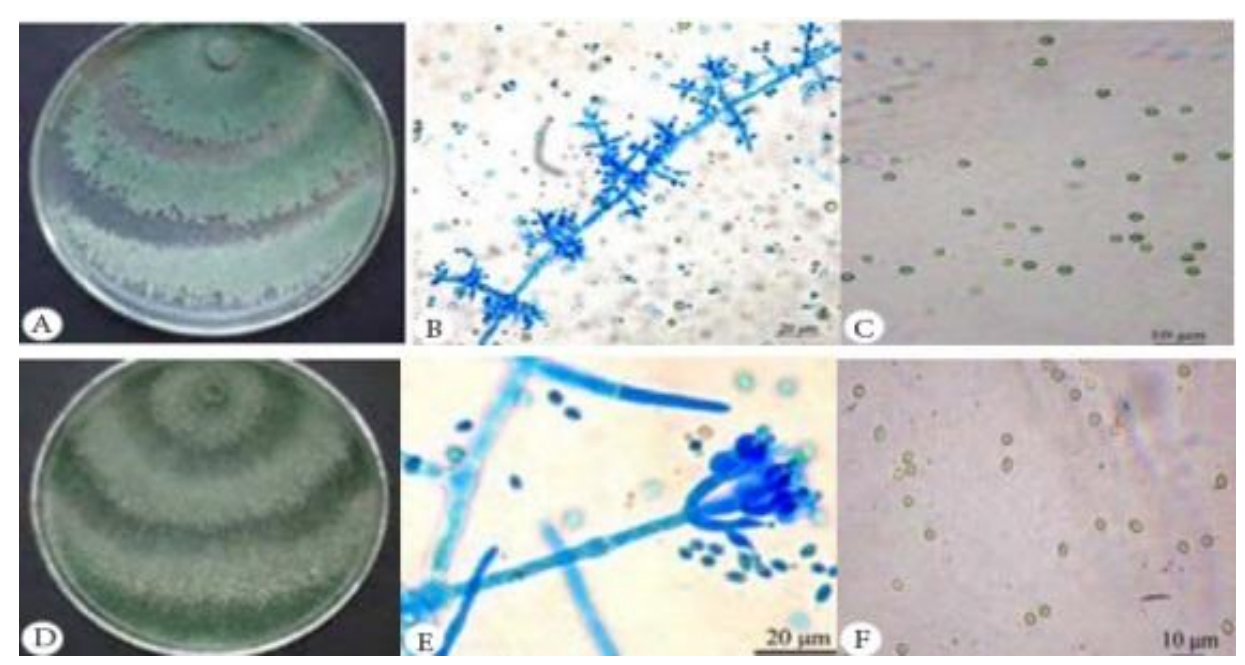

Fig. 1 Morphological characteristics of two species of Trichoderma. A and $D$ are colony morphology, $B$ and $E$ are Phialide shape, $C$ and $F$ are conidia shape [20].

\section{Artificial neural network design for recognition of Trichoderma}

In this section, after explaining neural network structure, qualitative characteristics will be quantified and dataset will be applied to the neural network.

\subsection{Structure selection of the network}

The network used for identifying fungus is a MLP (multilayer perceptron). Fig. 3 shows overall structure of the neural network. The network includes three layers: input layer, hidden layer and output layer. According to [11], one hidden layer is enough for solving classification problems by using artificial neural network. Hence, one layer was selected as hidden layer. In this paper, number of cells in input layer is 14 (according to 14 characteristics used for identification of Trichoderma), number of cells in output layer cells is 25 cells (according to 25 fungal species) and 20 neurons were selected in hidden layer. This number was selected by investigating network for different hidden neurons from 1 to 25 neurons. Fig. 4 shows mean square error of the network in terms of number of neurons in hidden layer. It is obvious that optimum number for hidden neurons is 20 . 

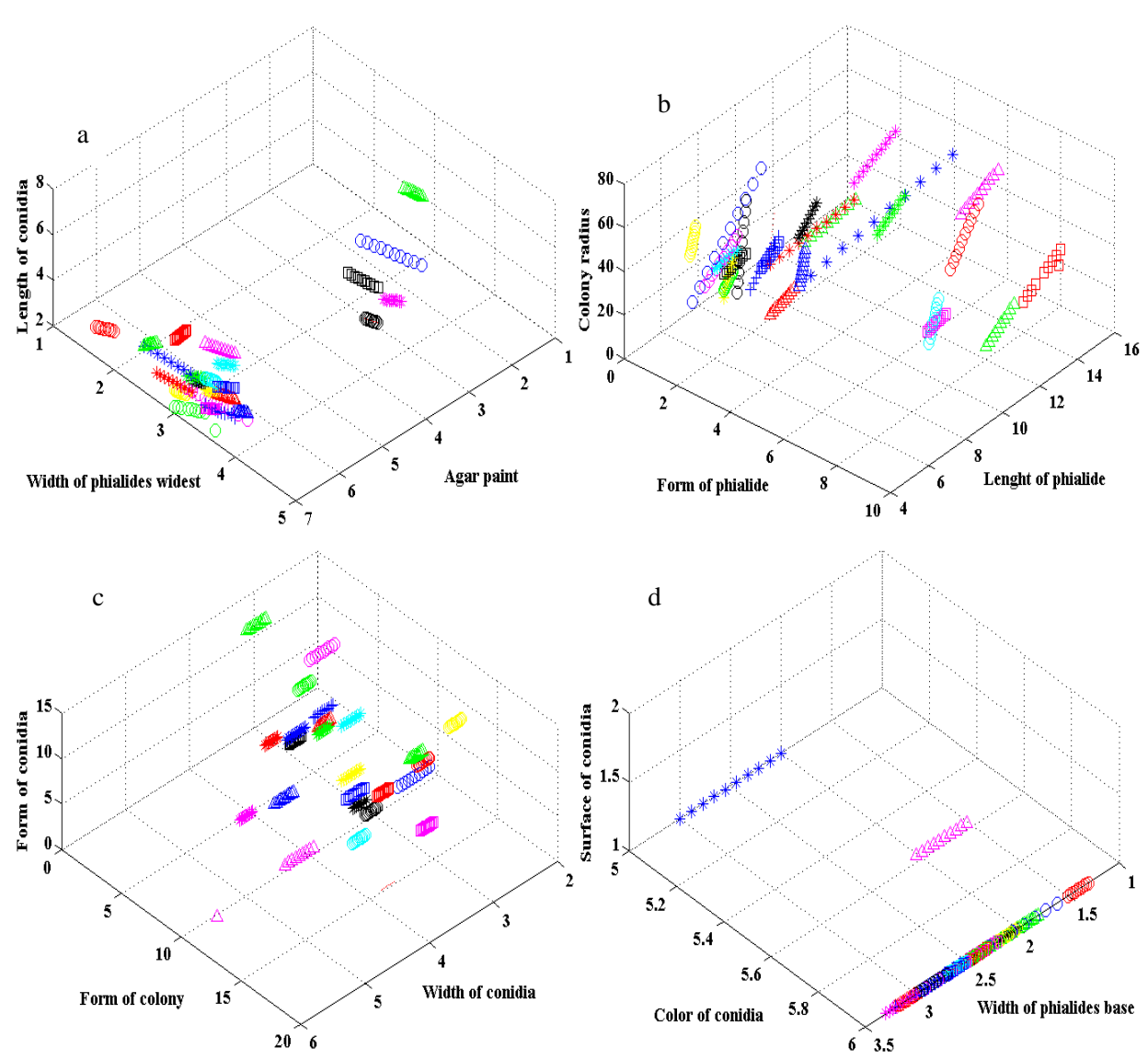

Fig. 2 Illustration of some features of Trichoderma in 3-dimensinal spaces shows that characteristics of this fungus are very close to each other. (a) Illustration of three characteristics: Agar paint, width at the widest sector of the phialide and length of the conidia regardless of other 11 features. (b) Illustration of three characteristics: form of the phialides, their length, and colony radius after three days regardless of other 11 features. (c) Illustration of three characteristics: width of conidia, their form and colony morphology regardless of other 11 features. (d) Illustration of three characteristics: width of phialide's base, conidia color and its surface regardless of other 11 features.

Back propagation algorithm was used for training the network where real outputs are compared with desired outputs and weights are adjusted according to error propagated back in the network. For $p$-th input pattern, error reference for all cells of output layer of the network is given by

$$
E_{p}=\frac{1}{2}\left(t^{p}-a^{p}\right)^{2}=\frac{1}{2} \sum_{j=1}^{\mathbf{s}_{1}}\left(t_{j}^{p}-a_{j}^{p}\right)^{2},
$$

where $t_{j}^{p}$ is target output for $p$-th input in $j$-th cell of the output layer, $a_{j}^{p}$ is 
produced real output for $p$-th input in $j$-th cell of the output layer and $\mathbf{s}_{1}$ is length of output vector. Overall error for $p$ models is obtained by

$$
E=\sum_{p=1}^{\mathbf{r}} E_{p}=\frac{1}{2} \sum_{p=1}^{\mathbf{r}} \sum_{j=1}^{\mathbf{s}_{1}}\left(t_{j}^{p}-a_{j}^{p}\right)^{2} .
$$

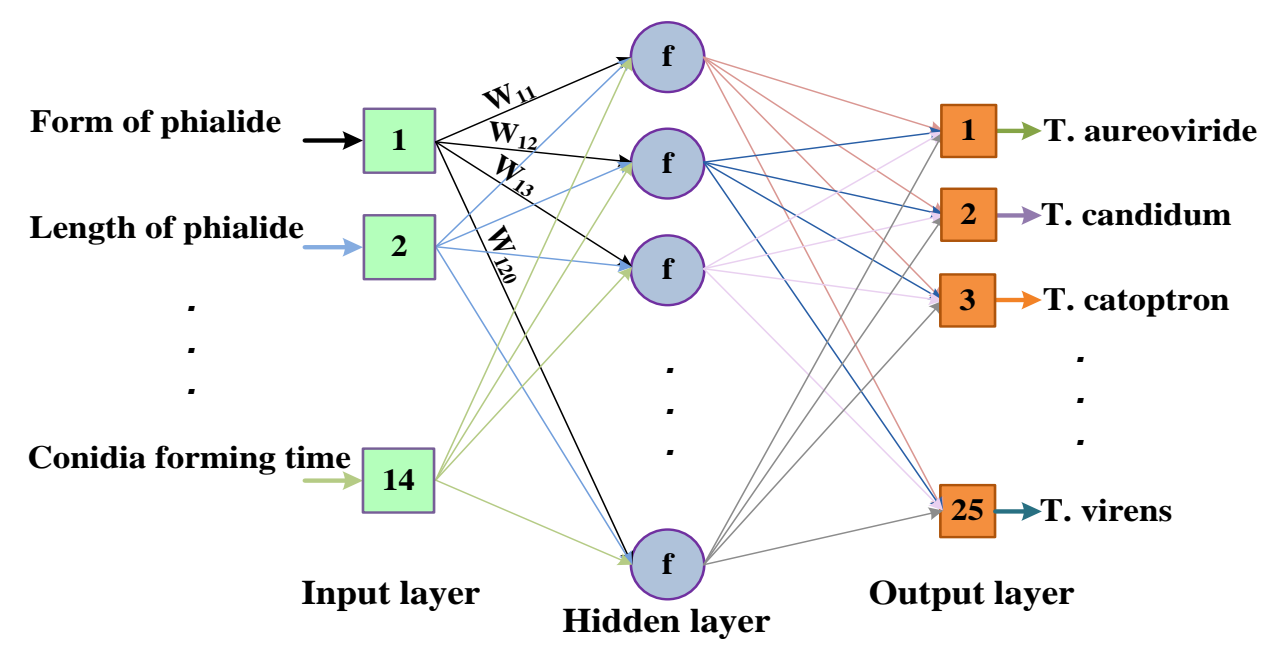

Fig. 3 Overall structure of MLP neural network employed for identification of different species of Trichoderma.

Weights are adjusted by gradient method to reduce cost function $E$ to minimum value. Rule of weight updating is given by

$$
w_{i j}(t+1)=\eta \Delta w_{i j}(t)+\alpha \Delta w_{i j}(t-1),
$$

where $\Delta w_{i j}(t)$ is equal to $-\left(\frac{\partial E_{p}}{\partial w_{i j(t)}}\right), \eta$ is learning rate which is selected in the range from 0.2 to $0.8, \alpha$ is instantaneous coefficient, $w_{i j}(t+1)$ is new weight, and $w_{i j}(t)$ is old weight. Bias vector is updated by

$$
b_{j}(t+1)=b_{j}(t)+\eta \Delta b_{j}(t)+\alpha \Delta b_{j}(t-1),
$$

where $\Delta b_{j}(t)$ is equal to $-\left(\frac{\partial E_{p}}{\partial b_{j}(t)}\right), b_{j}(t+1)$ is new bias, and $b_{j}(t)$ is old bias. Weights and biases are updated by Eqs. (3) and (4). Training process is stopped when overall error becomes smaller than a pre-specified threshold value or maximum number of training epochs is reached [13].

\subsection{Designing of the network}

In order to identify different species of Trichoderma, aforementioned MLP neural network should be trained using morphological characteristics. To this end, qualitative characteristics of the fungus should be quantified, first. Among the 
Neural Network World 2/2016, 155-173

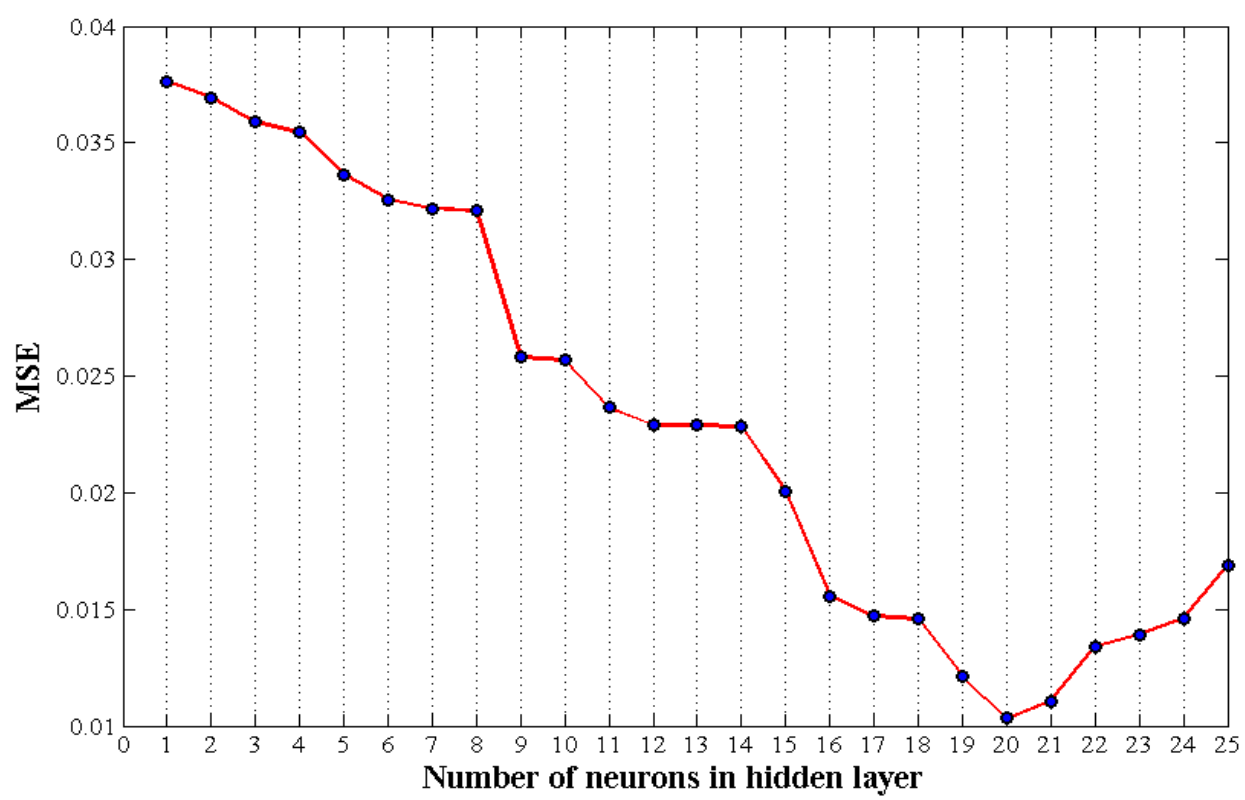

Fig. 4 Plot of MSE for selecting the number of neuron in hidden layer.

characteristics, only colors and shapes are qualitative. Colors are quantified according to color level as shown in Tab. I. Shapes of conidia, phialide and colony are labeled as Tabs. II, III, and IV, respectively. Surface of conidia is also labeled as Tab. V. The morphological characteristics of fungus are subsequently represented as 14-dimensional numerical vectors those are applied as input data to the network. This input vector is shown by

$$
P=\left[\begin{array}{l}
\text { Form of the phialide } \\
\text { Length of the phialide } \\
\text { Width at the widest sector of the phialide } \\
\text { Width of the phialide's base } \\
\text { Conidia color } \\
\text { Conidia surface } \\
\text { Form of the conidia } \\
\text { Length of the conidia } \\
\text { Width of the conidia } \\
\text { Form of the colony } \\
\text { Colony's forming time } \\
\text { Agar's paint } \\
\text { Colony radius at } 25^{\circ} \mathrm{C} \text { after } 3 \text { days } \\
\text { Conidia forming time on agar }
\end{array}\right]
$$

Target species, those are applied as desired outputs to the network, are also sorted in two first columns of Tab. VI. The artificial neural network has 25 outputs corresponding to 25 species. In a well-trained network, if input data are matched 


\begin{tabular}{lc}
\hline Color & Level \\
\hline Brownish & 1 \\
Yellowish or brownish & 2 \\
Light Yellow & 3 \\
Yellow & 4 \\
Pale green & 5 \\
Green & 6 \\
No pigment & 7 \\
\hline
\end{tabular}

Tab. I Level of colors.

\begin{tabular}{lc}
\hline Form of the Phialide & Label \\
\hline Tapering & 1 \\
Ampulliform & 2 \\
Ampulliform broader in middle \&conistricted in tip & 3 \\
Ampulliform to flask shaped & 4 \\
Flask-shaped or lagneiform, tapering uniformly from & 5 \\
base to tip, slender sometimes twisted \& short & 6 \\
Cylindrical & 7 \\
Cylindrical to subulate & 8 \\
Slender, tapering & 9 \\
Ampulliform somewhat hooked & \\
\hline
\end{tabular}

Tab. II Label of Phialide's form.

\begin{tabular}{lc}
\hline Form of the conidia & Label \\
\hline Globose to subglobose & 1 \\
Subglobose & 2 \\
Subglobose To Ellipsoidal & 3 \\
Subglobose to broadly ellipsoidal & 4 \\
Subglobose to ovoidal & 5 \\
Ellipsoidal & 6 \\
Broadly ellipsoidal to obovoid & 7 \\
Broad ellipsoidal & 8 \\
Ellipsoidal to oblong & 9 \\
Ellipsoidal rarely oblong & 10 \\
Obovoid to subglobose & 11 \\
Oblong to ellipsoidal & 12 \\
Clavate to ellipsoidal or subglobose & 13 \\
\hline
\end{tabular}

Tab. III Label of Conidia's form. 
to $i$-th species, then $i$-th output must be 1 (fired) and other outputs must be -1 (unfired). In training stage, this implication is exploited to determine desired output for any given input data. However, in validation stage where some unseen inputs are applied, outputs of the network would not be exactly 1 or -1 . The justification is proximity of characteristics of different species as it was explained in Section 3. After training, when features of an unknown fungus are applied to input of a network, a membership degree between -1 and 1 is generated at each output node. Maximum value of these membership functions, which is expected to be near 1 , will be interpreted as the species that the input data is more likely to belong to it.

In training process, initial weights of each layer are randomly selected and then tuned by back-propagation algorithm. After completing training process of neural network, weights remain fixed and the network is ready to be validated. After validation, the network can start its function mode and can be used as classification tool.

\begin{tabular}{lc}
\hline Form of the colony & Label \\
\hline Abundant aerial mycelium & 1 \\
Flat with highly aggregate compact & 2 \\
Cottony with conidiophores conidia around the point of inoculum & 3 \\
Flat, uniformly covered by conidia & 4 \\
Flat, aggregate pustules forming from the point of inoculum outwards, & 5 \\
conidia pale yellow & \\
Somewhat cottony with higher concentration of aerial hyphae near & 6 \\
edges of plate & \\
Flat, uniformly covered by few pale green conidia & 7 \\
Flat, with abundant conidia formed in highly aggregate concentric & 8 \\
ring & \\
Cottony & 9 \\
Flat with scant aerial mycelium & 10 \\
Flat, cottony & 11 \\
Somewhat cottony & 12 \\
Flat, with some aerial mycelium & 13 \\
Flat, with pustules forming towards the point of inoculum & 14 \\
Floccose with effuse conidiation & 15 \\
\hline
\end{tabular}

Tab. IV Label of Colony's form.

\begin{tabular}{ccc}
\hline Surface & Smooth & Smooth to slightly roughened \\
\hline Label & 1 & 2 \\
\hline
\end{tabular}

Tab. V Label of Surface of conidia. 
To design the network, 250 datasets (10 data sets for each species) are used which obtained from [7]. Among these 250 data, $60 \%$ of data sets are used for training network, $20 \%$ of datasets are used for network validation and remaining $20 \%$ are used for network testing.

\section{Classification results}

After designing network, it is required that the network is trained and tested by some dataset. In this section, the performance of the trained network is examined by two case studies.

\subsection{Training network}

While the artificial neural network is being trained, network weights and biases are updated at each time step. Training is continued until mean squared error (MSE) of the network is reduced to a threshold value. In order to avoid over-fitting in training, this threshold is selected to be MSE of validation datasets. As it can be seen in Fig. 5, MSE starts from a large value in the first epoch and decreases gradually. It means that network training process is in progress. The best network performance for validation datasets is obtained in epoch 10 with MSE of 0.01033 .

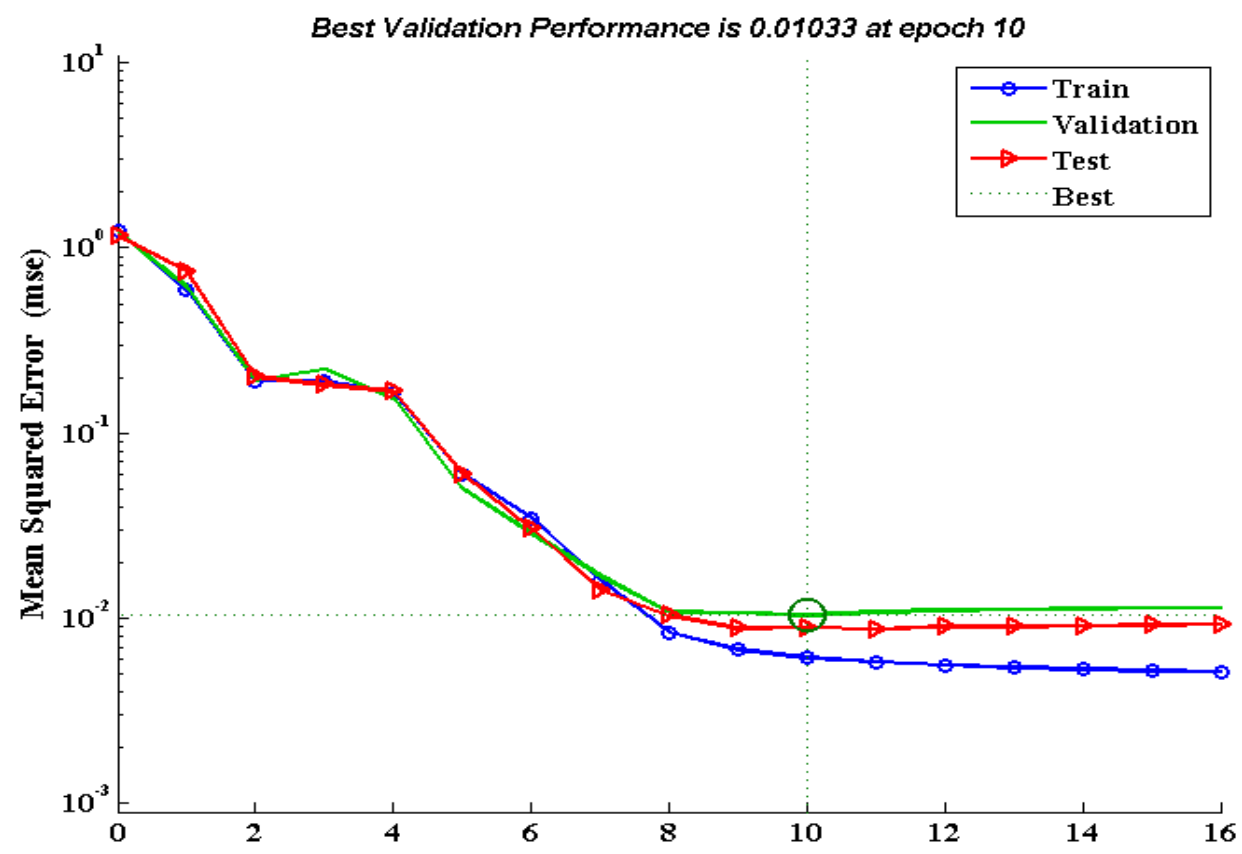

Fig. 5 Mean squared error of the network for 16 epochs.

Fig. 6 shows accuracy and matching of the network output with the expected output. This figure is related to network regression. Symbol $\bigcirc$ in this figure shows network output. In this figure, outputs less than 0.4 are mapped to 0 and outputs 

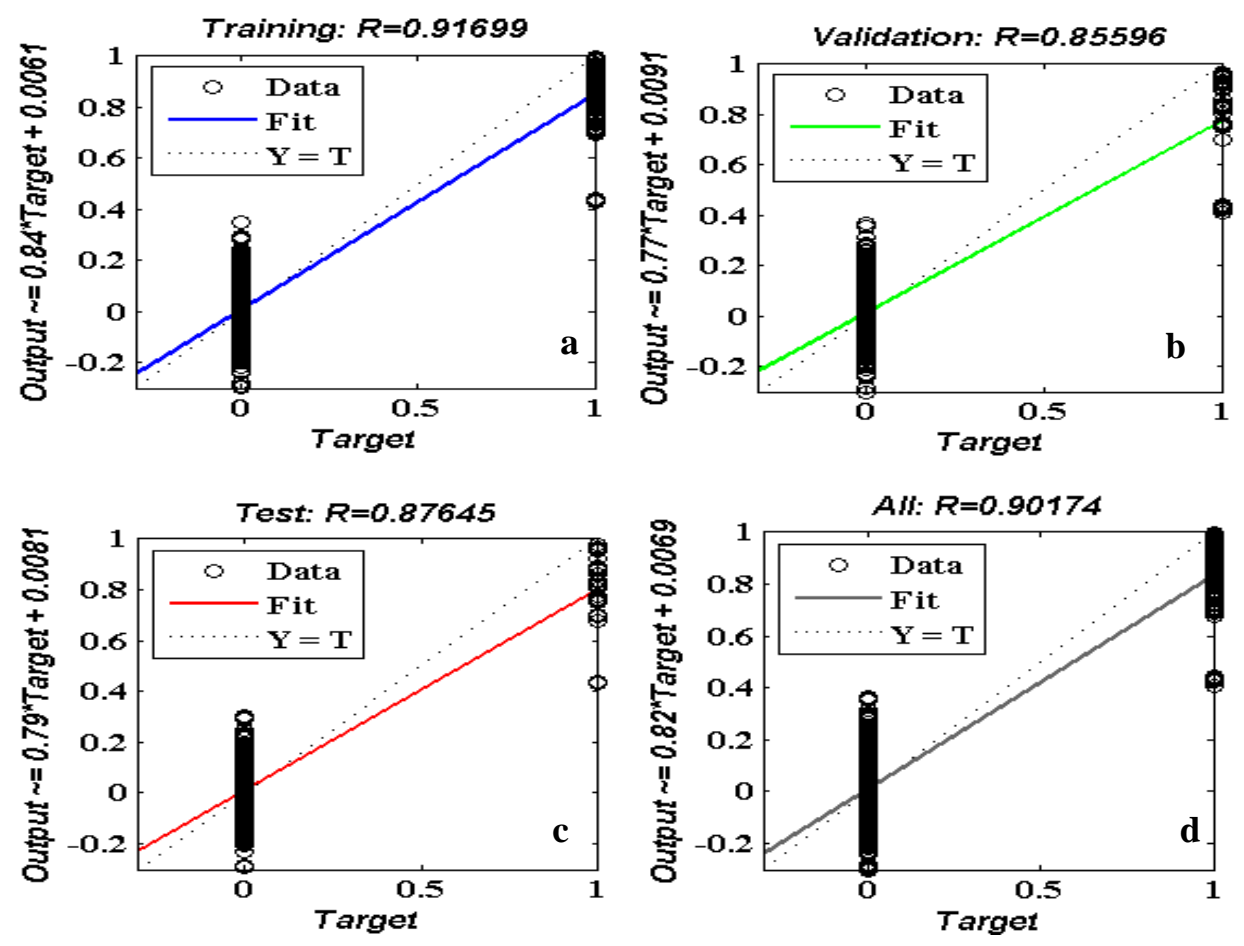

Fig. 6 Network regression graphs for datasets. (a) Network regression for training data. (b) Network regression for validation data. (c) Network regression for testing data. (d) Network regression for all data.

more than 0.4 are mapped to 1 . It is desired that all outputs are matched with the target value $(O=T)$ that corresponds to dotted lines in figures. The values obtained by the network are fitted by first order polynomials that correspond to colored lines. If the dotted lines are more matched with colored lines, value of $R$ will be high and there will be more matching between network output and expected output. $R=1$ means that outputs are exactly matched with targets.

From Figs. 6(a), 6(b), 6(c) it can be seen that for training, validation and testing data, regression accuracy are $91.699 \%, 85.596 \%, 87.645 \%$, respectively. Fig. 6(d) shows the regression accuracy of the network for all data is equal to $90.174 \%$.

\subsection{Network test}

As explained earlier, in output layer of the neural network, a membership between $[-1,+1]$ is generated for each input dataset. After training network, input data is applied to the network to investigate these values. Fig. 7 shows the diagram of the values that the neural network has assigned as membership degree to 250 input data. For each data in this figure, only one membership degree is close to 1. Maps of these points are shown on the lower plan in red. Corresponding values, assigned to 250 input data, are also shown in Tab. VI illustrating that 


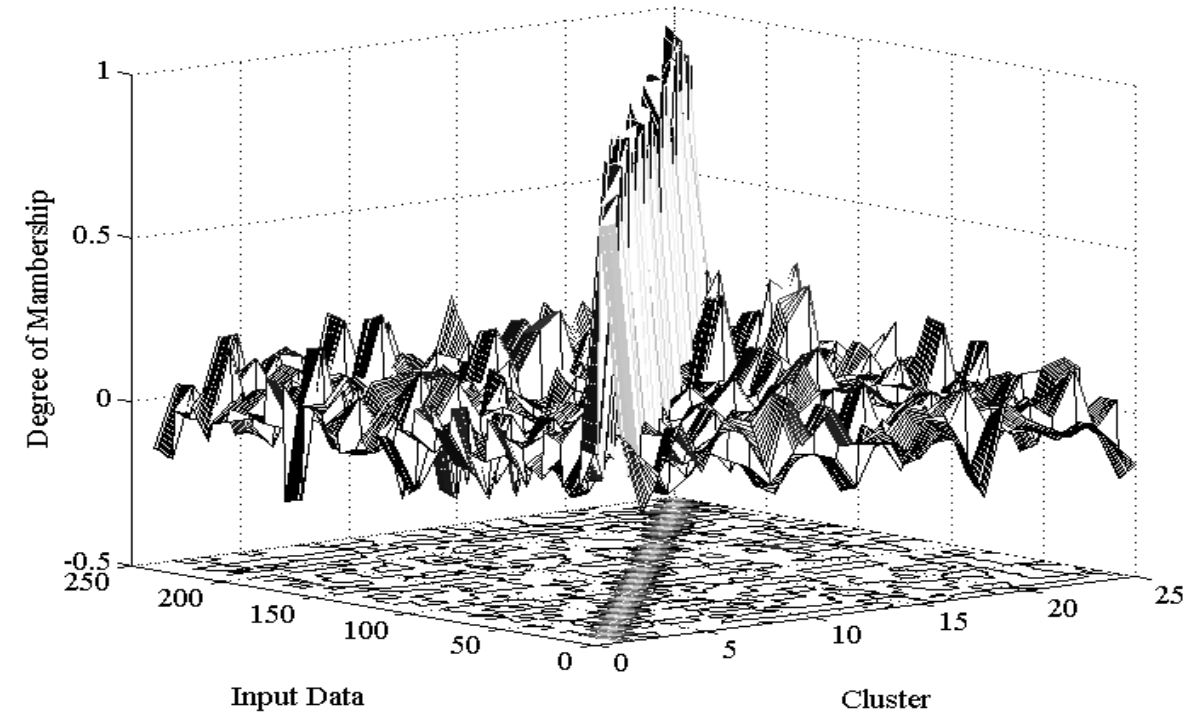

Fig. 7 Diagram of membership degree generated in output layer of the network corresponding to 250 input data. In the projection of the values on lower plane maximum membership degree for 250 species are shown by diagonal gray bar.

which input data corresponds to which class. As it can be seen the neural network could properly distinguish most of species by assigning their membership degree and it could identify input data with high accuracy.

\subsection{Case studies}

As two case studies, two species of Trichoderma of Fig. 1 were selected and the trained neural network was employed to identify them. Morphological characteristics of these two species were applied as input to the neural network and checked if the network could recognize them.

Case 1: In Fig. 1, morphological characteristics for the species in the top (subfigures A, B, C) are as the sequel:

- Radius of the colony after 3 days is $65 \mathrm{~mm}$.

- Colonies are somewhat cottony after 1 week (quantified as 12).

- Conidia are produced frequently and distributed in environment after 4 days; rear side of the colony is yellowish (quantified as 2).

- Phialides are ampulliform (quantified as 2), their length is $3.6-8 \mu \mathrm{m}$, their width at the widest point is $2.4-3.6$, and their width at the base is $1.2-2.7 \mu \mathrm{m}$.

Conidia are subglobose to ovoidal (quantified as 5), their surface is smooth (quantified as 1) and green (quantified as 6), their length is $2.3-3.4 \mu \mathrm{m}$, and their width is $2-2.5 \mu \mathrm{m}$. Then, for this species the input vector is created as 
$\left[\begin{array}{l}\text { Form of the phialide } \\ \text { Length of the phialide } \\ \text { Width at the widest sector of the phialide } \\ \text { Width of the phialide's base } \\ \text { Conidia color } \\ \text { Conidia surface } \\ \text { Form of the conidia } \\ \text { Length of the conidia } \\ \text { Width of the conidia } \\ \text { Form of the colony } \\ \text { Colony's forming time } \\ \text { Agar's paint }\end{array}\right]=\left[\begin{array}{c}2 \\ \text { Colony radius at } 25^{\circ} \mathrm{C} \text { after } 3 \text { days }\end{array}\right]=\left[\begin{array}{c}3 \\ \text { Conidia forming time on agar }\end{array}\right]=\left[\begin{array}{c}2 \\ 6 \\ 1 \\ 5 \\ 2.9 \\ 2.5 \\ 12 \\ 7 \\ 2 \\ 65 \\ 4\end{array}\right]$

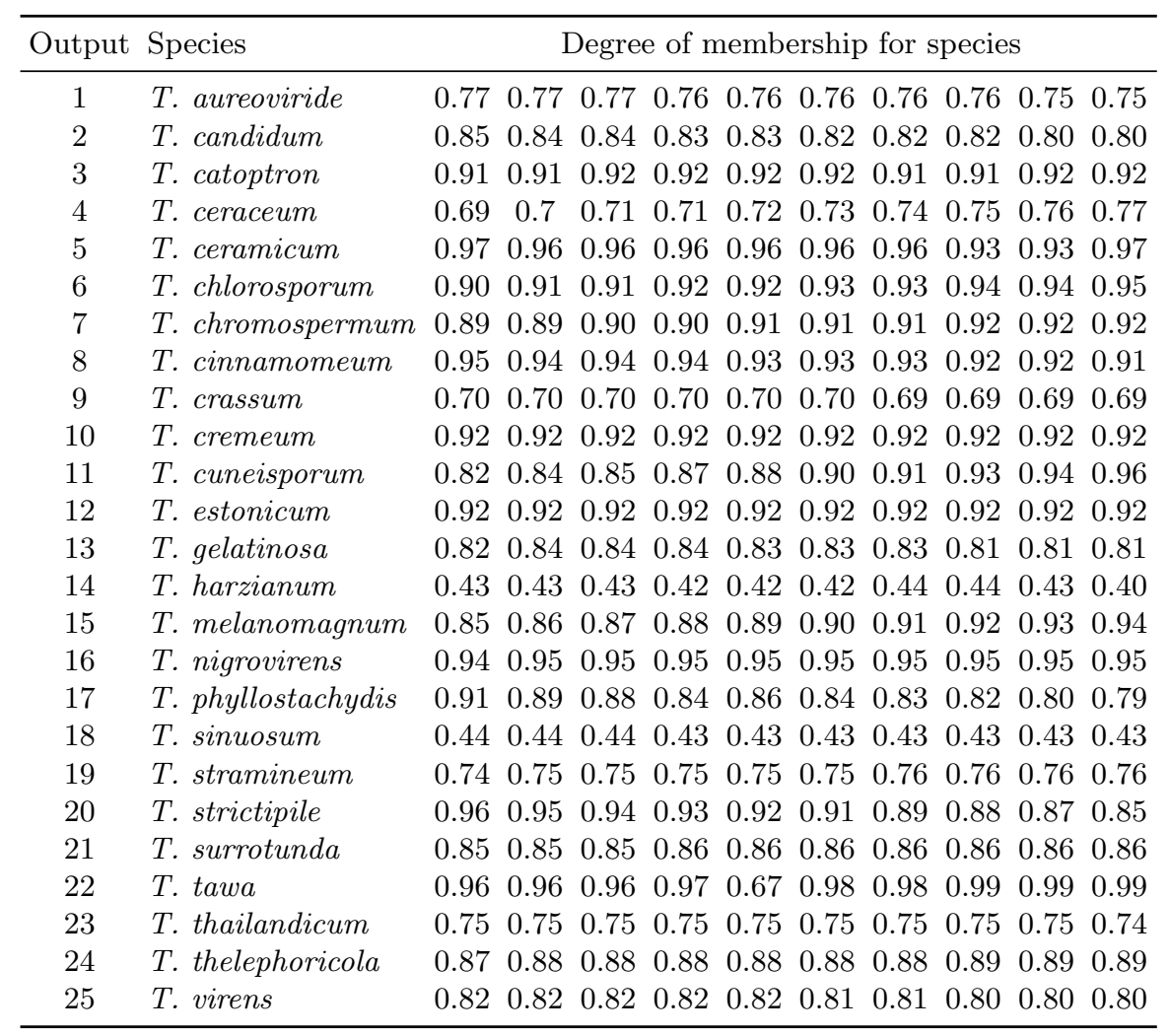

Tab. VI Maximum membership degree for 250 species of Trichoderma generated by the neural network. 
Case 2: In the same way, morphological characteristics for the species in the bottom of Fig. 1 (subfigures E, F, G) are as the following:

- Radius of the colony after 3 days is $43 \mathrm{~mm}$.

- Colonies are floccose with effuse conidiation after 1 week (quantified as 16).

- Conidia are produced frequently, distributed in environment after 1 week, and rear side of the colony is yellow (quantified as 4).

- Phialides are ampulliform (quantified as 2), their length is $5.5-12.5 \mu \mathrm{m}$, their width at the widest point is $2.7-5$, and width at the base is $1.7-2.5 \mu \mathrm{m}$.

- Conidia has smooth (quantified as 1) and green surface (quantified as 6), they are in subglobose form (quantified as 2), their length is $3.6-5.6 \mu \mathrm{m}$, and their width is $3-4 \mu \mathrm{m}$.

Then, for this species, the input vector is created as

$\left[\begin{array}{l}\text { Form of the phialide } \\ \text { Length of the phialide } \\ \text { Width at the widest sector of the phialide } \\ \text { Width of the phialide's base } \\ \text { Conidia color } \\ \text { Conidia surface } \\ \text { Form of the conidia } \\ \text { Length of the conidia } \\ \text { Width of the conidia } \\ \text { Form of the colony } \\ \text { Colony's forming time } \\ \text { Agar's paint } \\ \text { Colony radius at } 25^{\circ} \mathrm{C} \text { after } 3 \text { days } \\ \text { Conidia forming time on agar }\end{array}\right]=\left[\begin{array}{l}2 \\ 9 \\ 4 \\ 2.4 \\ 6 \\ 1 \\ 2 \\ 4.5 \\ 3.9 \\ 16 \\ 7 \\ 4 \\ 43 \\ 7\end{array}\right]$

After quantifying and creating input vectors, the data was applied to the neural network and membership degrees were generated by the network. These amounts are given numerically in Tab. VII. Maximum membership degree for species 1 (species in the top of Fig. 1) belongs to T. harzianum and maximum membership degree for species 2 (species in the bottom of Fig. 1) belongs to T. virens. By accommodating morphological characteristics with existing valid keys, it can be concluded that the network recognition for these species is correct.

\section{Conclusions}

Trichoderma species are important fungus in biological control of fungal plant disease and have crucial role in plant growth promotion. They also have several applications in industries. In the most of applications, it is important to distinguish among different species of Trichoderma and identify them. However, because of 
Neural Network World 2/2016, 155-173

\begin{tabular}{|c|c|c|c|}
\hline \multirow{2}{*}{ Output } & \multirow{2}{*}{ Species } & \multicolumn{2}{|c|}{ Membership } \\
\hline & & Data 1 & Data 2 \\
\hline 1 & T. aureoviride & -0.036 & -0.158 \\
\hline 2 & T. candidum & -0.02 & 0.067 \\
\hline 3 & T. catoptron & -0.144 & -0.088 \\
\hline 4 & T. ceraceum & -0.008 & 0.11 \\
\hline 5 & T. ceramicum & 0.099 & -0.04 \\
\hline 6 & T. chorosporum & -0.215 & 0.06 \\
\hline 7 & T. chromospermum & 0.127 & 0.11 \\
\hline 8 & T. cinnamomeum & -0.21 & -0.047 \\
\hline 9 & T. crissum & -0.025 & 0.19 \\
\hline 10 & T. cremeum & -0.002 & -0.0007 \\
\hline 11 & T. cuneisporum & -0.074 & 0.107 \\
\hline 12 & T. estonicum & 0.11 & -0.0001 \\
\hline 13 & T. gelatinosa & 0.137 & -0.077 \\
\hline 14 & T. harzianum & 0.423 & 0.076 \\
\hline 15 & T. melanomagnum & -0.058 & -0.086 \\
\hline 16 & T. nigrovirens & -0.047 & 0.026 \\
\hline 17 & T. phyllostachydis & 0.297 & 0.042 \\
\hline 18 & T. sinuosum & 0.029 & -0.068 \\
\hline 19 & T. stramineum & 0.055 & 0.135 \\
\hline 20 & T. strictipile & 0.225 & -0.022 \\
\hline 21 & T. surrotunda & 0.372 & 0.016 \\
\hline 22 & T. tawa & -0.12 & -0.063 \\
\hline 23 & T. thailandicum & 0.045 & -0.001 \\
\hline 24 & T. thelephoricola & -0.033 & -0.073 \\
\hline 25 & T. virens & 0.073 & 0.771 \\
\hline
\end{tabular}

Tab. VII Membership degree for test data corresponding to species of Fig. 1.

high number of species and their very similar morphological characteristics, manually distinguishing and identifying Trichoderma species is very difficult and time consuming.

In this paper, a multilayer perceptron neural network was trained by means of error back propagation algorithm in a supervisory approach to identify Trichoderma species. After training, the network could distinguish and identify 25 species. Statistical and validation analysis performed on 250 validation data and the results showed that the trained network exhibits accuracy of $100 \%$ in classification and accuracy of $90.174 \%$ in regression. As a case study, two species were considered and their data were quantified and applied to the network and it was shown that the network could successfully distinguish them. 


\section{References}

[1] BARNETT H.L., HUNTER B.B. Illustrated genera of imperfect fungi. Mycologia. 1972, 64(4), pp. 930-932, doi: 10.2307/3757954.

[2] Batta Y.A. Effect of Trichoderma harzianum Rifai formulated in invert emulsion on postharvest decay of apple blue mold. International journal of food microbiology. 2004, 69(3), pp. 281-288, doi: 10.1016/j.ijfoodmicro.2004.04.002.

[3] Batta Y.A. Postharvest Biological control of apple gray mold by Trichoderma harzianum Rifai formulated in invert emulsion. Crop Protection. 2004, 23(1), pp. 19-26, doi: 10.1016/ S0261-2194(03) 00163-7.

[4] Benitez T., Rincon A.M., Limon M.C., Codon A.C. Biocontrol mechanisms of Trichoderma strains [online]. International Microbiology. 2004, 7(4), pp. 249-260 [viewed 2016-05-04]. Available form: http://scielo.isciii.es/pdf/im/v7n4/Benitez.pdf

[5] Bouket A.C., Arzanlou, M., Babai-Ahari, A., FUNGID: a Web-based Identification Program for Phytophthora. International Journal of Computer Applications. 2012, 50(2), pp. 10-18, doi: 10.5120/7741-0793.

[6] Ceylana R., Özbaya Y., Karlikb B. A novel approach for classification of ECG arrhythmias: Type-2 fuzzy clustering neural network. Expert Systems with Applications. 2009, 36(3), pp. 6721-6726, doi: 10.1016/j.eswa.2008.08.028.

[7] CHAVERRI P., SAMUELS G.J. Hypocrea/Trichoderma (Ascomycota, Hypocreales, Hypocreaceae): species with green ascospores [online]. Studies in Mycology. 2003, 48(48), pp. 1-16 [viewed 2016-05-04]. Available from: https://www.researchgate. net/profile/Priscila_Chaverri/publication/259188197_HypocreaTrichoderma_ Ascomycota_Hypocreales_Hypocreaceae_species_with_green_ascospores_Stud_Mycol/ links/02e7e52a487bdbc37c000000.pdf

[8] CHITRA K., ANNADURAI G. Bioengineered silver nanobowls using Trichoderma viride and its antibacterial activity against gram-positive and gram-negative bacteria. Journal of Nanostructure in Chemistry. 2013, 3(9), pp. 1-7, doi: 10.?1186/?2193-8865-3-9.

[9] DE REspinis S., VOGEL G., BenAGli C., TONOlla M., PETRINI O., SAMUELS G.J. MALDI-TOF MS of Trichoderma: a model system for the identification of micro-fungi. Mycological Progress. 2010, 9(1), pp. 79-100, doi: 10.1007/s11557-009-0621-5.

[10] DRUZHININA I.S., KOPCHINSKIY A.G. An oligonucleotide barcode for species identification in Trichoderma and Hypocrea. Fungal Genetic and Biology. 2005, 42(10), pp. 813-28, doi: $10.1016 / \mathrm{j} . \mathrm{fgb} .2005 .06 .007$.

[11] GONZALEZ R.C., WOODS R.E. Digital Image Processing (chapter 12). 2nd ed. New Jersey: Prentice Hall, 2001.

[12] HAJIEGHRARI B., TORABI M., MOHAMMADI M.R., DAVARI M. Biological potential of some Iranian Trichoderma isolates in the control of soil borne plant pathogenic fungi. African Journal of Biotechnology. 2010, 71(8), pp. 788-799.

[13] HAYKIN S. Neural Networks: A Comprehensive Foundation. NewYork: Macmillan, 1994.

[14] HOITINK H.A.J., MODDEN L.V., DORRANCE A.E. Systemic resistance induced by Trichoderma spp.: interactions between the host, the pathogen, the biocontrol agent and soil organic matter quality. The American Phytopathology society Journal. 2006, 96(2), pp. 186-189, doi: 10.1094/PHYTO-96-0186.

[15] JADHAV S.M., NALBALWAR S.L., GHATOL A.A. Modular neural network based arrhythmia classification system using ECG signal data. International Journal of Information Technology and Knowledge Management. 2011, 4(1), pp. 205-209.

[16] Li X., Nie P., Qiu Z.J., He Y. Using wavelet transform and multi-class least square support vector machine in multi-spectral imaging classification of Chinese famous tea. Expert Systems with Applications. 2011, 38(9), pp. 11149-11159, doi: 10.1016/j.eswa.2011.02.160.

[17] Liu Z., Cheng F., Ying Y., Rao X. Identification of rice seed varieties using neural network. Journal of Zhejiang University Science B. 2005, 6(11), pp. 1095-1100, doi: 10.1631/jzus. 2005. B1095. 


\section{Neural Network World 2/2016, 155-173}

[18] Liu Z.Y., Wu H.F., Huang J.F. Application of neural networks to discriminate fungal infection levels in rice panicles using hyperspectral reflectance and principal components analysis. Computers and Electronics in Agriculture. 2010, 72(2), pp. 99-106, doi: 10.1016/j.compag. 2010.03 .003

[19] MANICKAVASAGAN A., SATHYA G., JAYAS D.S., WHITE N.D.G. Wheat class identification using monochrome images. Journal of Cereal Science. 2008, 47(3), pp. 518-527, doi: $10.1016 / j \cdot j c s .2007 .06 .008$.

[20] MORGAN A., BOBBY L., MORDUE J.E.M., MORRIS C.W. Evaluation of artificial neural networks for fungal identification, employing morphometric data from spores of Pestalotiopsis species. Cambridge Journals, Mycological Research. 1998, 102(08), pp. 975-984, doi: 10. $1017 /$ S0953756297005947.

[21] NAEIMI S., OKHOVVAT S.M., JAVAN NIKKHAH M., KREDICS L., KHOSRAVI V. Frequency and distribution of Trichoderma spp. in the paddy rice fields of Mazandaran province. Iranian Journal of Plant Protection Science. 2010, 40(2), pp. 79-91.

[22] NAUMANN A. A novel procedure for strain classification of fungal mycelium by cluster and artificial neural network analysis of Fourier transform infrared (FTIR) spectra. Analyst. 2009, 134(6), 1215-1223, doi: 10.1039/b821286d.

[23] NEILSON H.F., STEWART T.M. A multi-access lucid key to common plant pathogenic fungi causing diseases of temperate crops. New Zealand Plant Protection Society. 2003, 56, pp. $100-102$.

[24] NILSSON T., BASSANI M.R., LARSEN T.O., MONTANARELLA L. Classification of species in the genus Penicillium by Curie point pyrolysis/mass spectrometry followed by multivariate analysis and artificial neural networks. Journal of mass spectrometry. 1996, 31(12), pp. 1422-1428, doi: 10.1002/(SICI) 1096-9888.

[25] OU Y.Y., CHEN S.A., CHANG Y.M., VELMURUGAN D., FUKUI K., MICHAEL GROMIHA M. Identification of efflux proteins using efficient radial basis function networks with position-specific scoring matrices and biochemical properties. Proteins: Structure, Function, and Bioinformatics. 2013, 81(9), pp. 1634-1643, doi: 10.1002/prot. 24322.

[26] OUSLEY M.A., LYNCH J.M. Effect of Trichoderma on plant growth: A balance between inhibition and growth promotion. Microbial Ecology. 1993, 26(3), pp. 277-285, doi: 10.1007/ BF00176959.

[27] OZBAY Y., KARLIK B. A recognition of ECG arrhythmias using artificial neural networks In: Proceedings of the 23rd Annual Conference of the Engineering in Medicine and Biology Society. IEEE, 2001, 2, pp. 1680-1683, doi: 10.1109/IEMBS.2001.1020538.

[28] OZBAYA Y., CEYLANA R., KARLIK B. A fuzzy clustering neural network architecture for classification of ECG arrhythmias. Computers in Biology and Medicine. 2006, 36(4), pp. 376-388, doi: 10.1016/j.compbiomed.2005.01.006.

[29] OZBAYA Y., CEYLANA R., KARLIK B. Integration of type-2 fuzzy clustering and wavelet transform in a neural network based ECG classifier. Expert Systems with Applications. 2011, 38(1), pp. 1004-1010, doi: 10.1016/j.eswa.2010.07.118.

[30] RIFAI M.A. Revision of the genus Trichoderma. Mycological Papers. 1969.

[31] CHENARI BOUKET A., ARZANLOU M., BABAI-AHARI A., KHODABANDE A. FUNGID: a Web-based Identification Program for Phytophthora. International Journal of Computer Applications. 2012, 50(2), pp. 10-18, doi: 10.5120/7741-0793.

[32] RINU K., SATI P., PANDEY A. Trichoderma gamsii (NFCCI 2177): A newly isolated endophytic, psychrotolerant, plant growth promoting, and antagonistic fungal strain. Journal of Basic Microbiology. 2014, 54(5), pp. 408-417, doi: 10.1002/jobm.201200579.

[33] ROUGHANIAN M., ZAFARI D., AMINI J., ABDOLLAHZADEH J. Identification of Trichoderma spp. from West Iran. Indian Phytopathology. 2013, 66(2), pp. 186-189.

[34] SUTTON B.C. The Coelomycetes, Fungi imperfecti with pycnidia, acervuli and stromata. Commonwealth Mycological Institute, Kew, Surrey, UK, 1980. 
[35] TAHIR A.R., NEETHIRAJAN S., JAYAS D.S., SHAHIN M.A., SYMONS S.J., WHITE N.D.G. Evaluation of the effect of moisture content on cereal grains by digital image analysis. Food Research International. 2007, 40(9), pp. 1140-1145, doi: 10.1016/j.foodres .2007.06. 009.

[36] TAYLOR J.W., JACOBSON D.J., KROKEN S., KASUGA T., GEISER D.M., HIBBETT D.S., FISHER M.C. Phylogenetic species recognition and species concepts in Fungi. Fungal Genetic and Biology. 2000, 31(1), pp. 21-32, doi: 10.1006/fgbi.

[37] TRIPATHI R.M., GUPTA R.K., SHRIVASTAV A., SINGH M.P., SHRIVASTAV B.R., SINGH P. Trichoderma koningii assisted biogenic synthesis of silver nanoparticles and evaluation of their antibacterial activity. Advances in Natural Sciences: Nanoscience and Nanotechnology. 2013, 4(3), pp. 1-5, doi: 10.1088/2043-6262/4/3/035005.

[38] VOLPATO V., ADELFIO A., POLLASTRI G. Accurate prediction of protein enzymatic class by N-to-1 Neural Networks. BMC bioinformatics. 2013, 14(Suppl 1):S11, doi: 10.1186/ 1471-2105-14-S1-S11.

[39] ZAPOTOCZNY P. Discrimination of wheat grain varieties using image analysis and neural networks. Part I. Single kernel texture. Journal of Cereal Science. 2011, 54(1), pp. 60-68, doi: $10.1016 / j . j c s .2011 .02 .012$.

[40] ZHU Y., WANG Z., ZHOU J., WANG Z. Bacteria classification using Neural Network. In Proceedings of the 6th International Conference on Natural Computation, Yantai, Shandong, China. 2010, 3, pp. 1199-1203, doi: 10.1109/ICNC.2010.5583645. 